Supporting Information

\title{
Design Rules for Efficient Charge Transfer in Metal-Organic Framework Films: The Pore Size Effect
}

Meng Cai, Quentin Loague, and Amanda J. Morris*

Department of Chemistry, Virginia Polytechnic Institute and State University, 800 W. Campus Drive Blacksburg, Virginia 24061, United States

*To whom correspondence should be addressed.

E-mail: ajmorris@vt.edu.

Table of Contents

\begin{tabular}{|c|l|c|}
\hline Section S1 & Experimental methods & $\mathrm{S}-2$ \\
\hline Section S2 & Quantification of Fc-COOH in Fc-MOFs & $\mathrm{S}-8$ \\
\hline Section S3 & $\mathrm{N}_{2}$ sorption isotherm studies & $\mathrm{S}-10$ \\
\hline Section S4 & Scanning electron microscopy (SEM) images & $\mathrm{S}-12$ \\
\hline Section S5 & Scan rate dependence studies & $\mathrm{S}-14$ \\
\hline Section S6 & Quantification of $D_{e}$ and $D_{i}$ & $\mathrm{~S}-16$ \\
\hline Section S7 & Calculation of $D_{a p p}$ based on Anson plots & $\mathrm{S}-23$ \\
\hline Section S8 & References & $\mathrm{S}-26$ \\
\hline
\end{tabular}


Section S1. Experimental methods

Synthesis of 1,3,6,8-tetrakis(p-benzoic acid)pyrene (TBAPy) ligand for NU-1000 MOF

TBAPy was synthesized according to a previous report. ${ }^{1}$ A solution of 4(methoxycarbonyl)phenyl boronic acid (2.5 g, $16.5 \mathrm{mmol})$, 1,3,6,8-tetrabromopyrene (1.43 g, $2.7 \mathrm{mmol})$, palladium tetrakis(triphenylphosphine) (0.05 g, $0.05 \mathrm{mmol})$, and potassium carbonate $(3 \mathrm{~g}, 22 \mathrm{mmol})$ in dry dioxane $(20 \mathrm{~mL})$ was purged with nitrogen for $20 \mathrm{~min}$, and then stirred under nitrogen under reflux for $72 \mathrm{~h}$. After cooling down to room temperature, the mixture was condensed by rotary evaporation. The condensed mixture was then poured into a solution $(120 \mathrm{~mL})$ containing concentrated hydrochloric acid $(37 \%$ $\mathrm{HCl}$ ) and ice (w/w 1:3). The organic phase was extracted with $\mathrm{CHCl}_{3}$ (three times; $50 \mathrm{~mL}$ each time) and dried over magnesium sulfate. After removal of the solvent, the resulting solid was boiled in tetrahydrofuran (THF) for $2 \mathrm{~h}$, and insoluble material was collected by filtration to yield 1,3,6,8-tetrakis(4-methoxycarbonyl)phenyl pyrene.

To a solution of 1,3,6,8-tetrakis(4-methoxycarbonyl)phenyl pyrene ( $0.4 \mathrm{~g})$ in 40 $\mathrm{mL}$ of a mixture of THF/dioxane/water (5:2:2), $10 \mathrm{~mL}$ of $1 \mathrm{M} \mathrm{NaOH}$ solution were added under stirring. The mixture was heated under reflux overnight until a clear yellow solution was obtained. The solvent was removed under reduced pressure, and the residue was dissolved in $25 \mathrm{~mL}$ of water and stirred for another $2 \mathrm{~h}$. Concentrated $\mathrm{HCl}$ was then used to adjust the $\mathrm{pH}$ of the solution to 2 . The yellow precipitate was collected by filtration and washed with water, $\mathrm{HCl}$ solution $(1 \mathrm{M})$, and diethyl ether. After drying under vacuum, $0.36 \mathrm{~g}(86 \%)$ of 1,3,6,8-tetrakis(p-benzoic acid)pyrene (TBAPy) was obtained. ${ }^{1} \mathrm{H}$ NMR (400 MHz, d6-DMSO): $\delta 13.1$ (s, 4H), 8.20 (s, 4H), 8.15 (d, 8H), 8.07 (s, 2H), $7.86(\mathrm{~d}, 8 \mathrm{H})$ 


\section{Synthesis of 1,3,6,8-tetra(6-carboxynaphthalen-2-yl)pyrene (TCNPy) ligand for NU-1003}

Prior to the synthesis of TCNPy, methyl 6-(pinacolboryl)-2-naphthoate was synthesized following a slightly modified procedure based on previous literature. ${ }^{2}$ A mixture of methyl 6-bromo-2-naphtoate $(2.14 \mathrm{~g}, 8.10 \mathrm{mmol}), \mathrm{B}_{2} \mathrm{Pin}_{2}(2.26 \mathrm{~g}, 8.89 \mathrm{mmol})$, potassium acetate $(2.38 \mathrm{~g}, 24.25 \mathrm{mmol}), \mathrm{PdCl}_{2}(\mathrm{dppf}) \cdot \mathrm{CH}_{2} \mathrm{Cl}_{2}(0.20 \mathrm{~g}, 0.24 \mathrm{mmol})$ was dissolved in dry DMSO $(50 \mathrm{~mL})$. The reaction mixture was stirred under nitrogen flow at $80{ }^{\circ} \mathrm{C}$ overnight. After cooling down to room temperature, the mixture was added to water (200 $\mathrm{mL}$ ) under stirring. The resulting precipitate was collected by filtration and washed with water. The precipitate was then dissolved in a mixture of dichloromethane (DCM, $50 \mathrm{~mL}$ ) and water $(50 \mathrm{~mL})$. The organic phase was collected, and the aqueous phase was extracted several times with DCM. The combined organic phase was washed with brine $(20 \mathrm{~mL})$ and dried over magnesium sulfate. The solvent was removed by rotary evaporation to yield methyl 6-(pinacolboryl)-2-naphthoate (1.98 g, 78\%), which was used for following synthesis without further purification.

A solution of 1,3,6,8-tetrabromopyrene (0.71 g, $1.4 \mathrm{mmol})$, methyl 6-(pinacolboryl)-2naphthoate $(1.90 \mathrm{~g}, 6.1 \mathrm{mmol})$, potassium carbonate $(1.53 \mathrm{~g}, 11.1 \mathrm{mmol})$, and palladium tetrakis(triphenylphosphine) $(0.08 \mathrm{~g}, 0.07 \mathrm{mmol})$ in dry dioxane $(35 \mathrm{~mL})$ was heated under nitrogen at $90{ }^{\circ} \mathrm{C}$ for 3 days. After cooling down to room temperature, $35 \mathrm{~mL}$ of water was added to the mixture. The insoluble material was collected by filtration, washed with water and acetone, and boiled in chloroform and filtered hot. The filtrate was collected and the solvent was removed under vacuum. The crude was dispersed in silica gel and purified by column chromatography (5\%-40\% ethyl acetate in DCM) to yield a yellow powder (the precursor of TCNPy). 
The precursor $(0.2 \mathrm{~g})$ was added to $10 \mathrm{~mL}$ of dioxane, and a solution of potassium hydroxide ( $0.12 \mathrm{~g}$ in $10 \mathrm{~mL}$ water) was added under stirring. The mixture was heated under reflux overnight until a clear yellow solution was obtained. After cooling down to room temperature, the solvent was removed by rotary evaporation. The resulting material was dissolved in $20 \mathrm{~mL}$ of water and acidified by concentrated $\mathrm{HCl}$ to $\mathrm{pH} 1$. The resulting precipitate was collected by centrifugation and washed with water.

\section{Synthesis of MOF-808}

To a glass jar $(60 \mathrm{~mL}), 0.16 \mathrm{~g}(0.5 \mathrm{mmol})$ of $\mathrm{ZrOCl}_{2} \cdot 8 \mathrm{H}_{2} \mathrm{O}$ and $0.11 \mathrm{~g}(0.5 \mathrm{mmol})$ of $1,3,5-$ benzenetricarboxylic acid were dispersed in a mixture of DMF/formic acid $(20 / 20 \mathrm{~mL})$. The reaction mixture was heated at $130{ }^{\circ} \mathrm{C}$ in oven for 2 days. The resulting white powders were collected by centrifugation, washed with DMF and acetone, and finally dried under vacuum.

\section{Synthesis of NU-1000}

To a 6-dram vial, $0.104 \mathrm{~g}$ of $\mathrm{ZrOCl}_{2} \cdot 8 \mathrm{H}_{2} \mathrm{O}$ and $2.7 \mathrm{~g}$ of benzoic acid were dissolved in $8 \mathrm{~mL}$ of DMF, and heated at $80{ }^{\circ} \mathrm{C}$ for $1 \mathrm{~h}$. After cooling down to room temperature, $40 \mathrm{mg}$ of TBAPy ligand was added and sonicated for $20 \mathrm{~min}$. The vial was then placed in an oven at $120^{\circ} \mathrm{C}$ for 2 days. The resulting yellow powders were collected by centrifugation, washed with DMF and acetone, and dried under vacuum at $120^{\circ} \mathrm{C}$.

\section{Synthesis of NU-1003}

To a 6-dram vial, $0.04 \mathrm{~g}$ of $\mathrm{ZrOCl}_{2} \cdot 8 \mathrm{H}_{2} \mathrm{O}$ and $0.625 \mathrm{~g}$ of benzoic acid were dissolved in $10 \mathrm{~mL}$ of DMF, and heated at $80^{\circ} \mathrm{C}$ for $1 \mathrm{~h}$. After cooling down to room temperature, $16 \mathrm{mg}$ of TCNPy in $10 \mathrm{~mL}$ of DMF and $300 \mu \mathrm{L}$ of trifluoroacetic acid was added. The vial was then placed in an 
oven at $120^{\circ} \mathrm{C}$ for $3 \mathrm{~h}$. The resulting yellow powders were collected by centrifugation, washed with DMF and acetone, and dried under vacuum at $120^{\circ} \mathrm{C}$.

\section{Synthesis of tetrabutylammonium tetrakis(pentafluorophenyl)borate (TBATFAB), $\left[\left(\mathrm{C}_{4} \mathrm{H}_{9}\right)_{4} \mathrm{~N}\left[\mathrm{~B}\left(\mathrm{C}_{6} \mathrm{~F}_{5}\right)_{4}\right]\right.$}

TBATFAB was synthesized following a previous procedure. ${ }^{3} 10.00 \mathrm{~g}$ of $\mathrm{Li}\left[\mathrm{B}\left(\mathrm{C}_{6} \mathrm{~F}_{5}\right)_{4}\right] / \mathrm{n} \cdot \mathrm{Et}_{2} \mathrm{O}$ was dissolved in $20 \mathrm{~mL}$ of methanol. To this solution, another $10 \mathrm{~mL}$ of methanol with $5.10 \mathrm{~g}$ (15.80 mmol) of $\left[\mathrm{N}_{(}\left(\mathrm{C}_{4} \mathrm{H}_{9}\right)_{4}\right] \mathrm{Br}$ was added dropwise over a 15-min period under stirring. Afterwards, $3 \mathrm{~mL}$ of water was added dropwise. The solution was stored at $-18{ }^{\circ} \mathrm{C}$ overnight. The resulting precipitate was collected by filtration and washed with $10 \mathrm{~mL}$ of cold methanol. The precipitate was dissolved in excess $(30 \mathrm{~mL})$ dichloromethane and dried over $\mathrm{MgSO}_{4}$. The solvent was removed by rotary evaporation to yield an off-white crude product (9.56 g, 94\%).

For purification, the crude was dissolved in $11 \mathrm{~mL}$ of dichloromethane. Diethyl ether was then added dropwise to the DCM solution until precipitate was formed. After being stored at $-18{ }^{\circ} \mathrm{C}$ overnight, the resulting precipitate was filtered and washed with hexanes to obtain pure white crystalline.

\section{Solvent Assisted Ligand Incorporation (SALI)}

Ferrocene carboxylic acid was incorporated into three MOFs via SALI following a previously reported procedure. ${ }^{4}$ In a typical experiment, $0.027 \mathrm{mmol}$ of MOF was heated in $2.4 \mathrm{~mL}$ of $0.1 \mathrm{M}$ ferrocene carboxylic acid in DMF at $60{ }^{\circ} \mathrm{C}$ for $20 \mathrm{~h}$ with occasional swirling. The resulting ferrocene-loaded MOF powders were collected by centrifugation, washed with DMF, acetone and diethyl ether, and finally dried under vacuum. To determine the loading level of ferrocene, 
several drops of concentrated $\mathrm{H}_{2} \mathrm{SO}_{4}$ were added to completely digest $\sim 5 \mathrm{mg}$ of MOF samples, and d6-DMSO was added. The solution was sonicated and then ready for NMR.

\section{Electrophoretic deposition of MOF thin films}

MOF thin films were prepared by electrophoretic deposition following previous work. ${ }^{4} 10 \mathrm{mg}$ of ferrocene loaded MOF was dispersed in $25 \mathrm{~mL}$ of toluene in a glass jar by sonication. The solution was stirred throughout the electrophoresis. Two identical FTO slides that were $1 \mathrm{~cm}$ spaced with the conductive sides facing each other were submerged in the solution. A constant voltage of $130 \mathrm{~V}$ was applied to the FTO slides for $3 \mathrm{~h}$. Consequently, ferrocene loaded MOF thin films deposited on FTO slides were made.

\section{SEM}

Scanning electron microscopy (SEM) images were collected using a Leo/Zeiss 1550 fieldemission scanning electron microscope.

\section{PXRD}

Powder X-ray Diffraction (PXRD) patterns were obtained with Rigaku Miniflex instrument $(\mathrm{Cu}$ $\mathrm{K} \alpha, \lambda=1.5418 \AA)$.

\section{$\mathbf{N}_{2}$ sorption studies}

The $\mathrm{N}_{2}$ sorption isotherms were collected on a Quantachrome Autosorb-1, and the BrunauerEmmett-Teller (BET) specific surface area was calculated by following a reported method. ${ }^{5}$

\section{Electrochemical measurements}

Electrochemical measurements were carried out with an EC epsilon potentiostat (BASi) using a three-electrode setup. A Fc-MOF film modified FTO, a platinum mesh and a non-aqueous 
$\mathrm{Ag} / \mathrm{Ag}^{+}$reference electrode were used as the working, counter and reference electrodes, respectively. Anhydrous MeCN containing $0.1 \mathrm{M} \mathrm{TBAPF}_{6}$ or TBATFAB was used as electrolyte. All potential values mentioned in text or presented in figures were converted to be versus ferrocene/ferrocenium $\left(\mathrm{Fc} / \mathrm{Fc}^{+}\right)$. 


\section{Section S2. Quantification of Fc-COOH in Fc-MOFs}

Quantification of Fc-COOH in each Fc-MOF was achieved by ${ }^{1} \mathrm{H}$ NMR studies following previous procedure. ${ }^{6}$ For each Fc-MOF sample, $\sim 3 \mathrm{mg}$ of Fc-MOF powder was digested by $\sim 5$ drops of concentrated $\mathrm{H}_{2} \mathrm{SO}_{4}$ solution. Afterwards, $\sim 600 \mu \mathrm{L}$ of DMSO-d6 was added to the solution and sonicated for $5 \mathrm{~min}$ to obtain a uniform solution. Quantitative ${ }^{1} \mathrm{H}$ NMR was performed on the prepared solution.

Proton peaks corresponding to the ligands in MOF-808 (top), NU-1000 (middle), and NU-1003 (bottom) are labeled in Figure S1. There are 3, 22, and 30 protons (excluding protons on carboxylic acid groups) respectively in one ligand molecule of MOF-808, NU-1000, and NU1003. The integrations are therefore normalized to corresponding number of protons. There are 9 protons (three proton groups) on one $\mathrm{Fc}$ molecule (excluding the proton on the carboxylic acid). If the $\mathrm{Fc} /$ ligand ratio is $1: 1$, the integration of $\mathrm{Fc}$ should be 9 . Therefore, the actual ratio of $\mathrm{Fc}$ vs ligand should be $\frac{F c \text { integration }}{9}$. For MOF-808, NU-1000, and NU-1003, the Fc/ligand ratios are $0.54,0.53$, and 0.54 , respectively. For all three MOFs, the node/ligand ratio is 1:2. Therefore, $\mathrm{Fc} /$ node ratios are $1.09,1.06$, and 1.09 , respectively. Generally, there is one $\mathrm{Fc}$ per node for all three Fc-MOFs. 


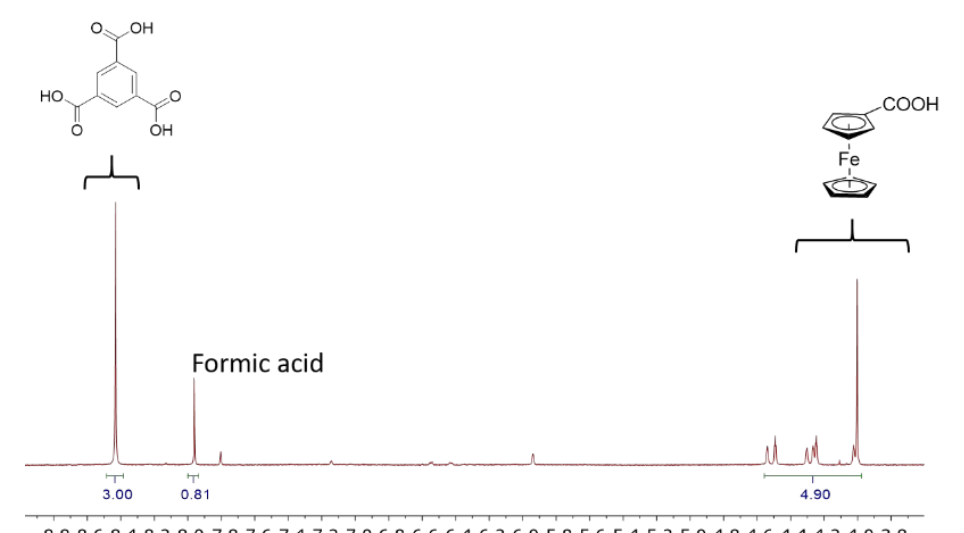

8.88 .68 .48 .28 .07 .87 .67 .47 .27 .06 .86 .66 .46 .26 .05 .8
chemical shift (ppm)
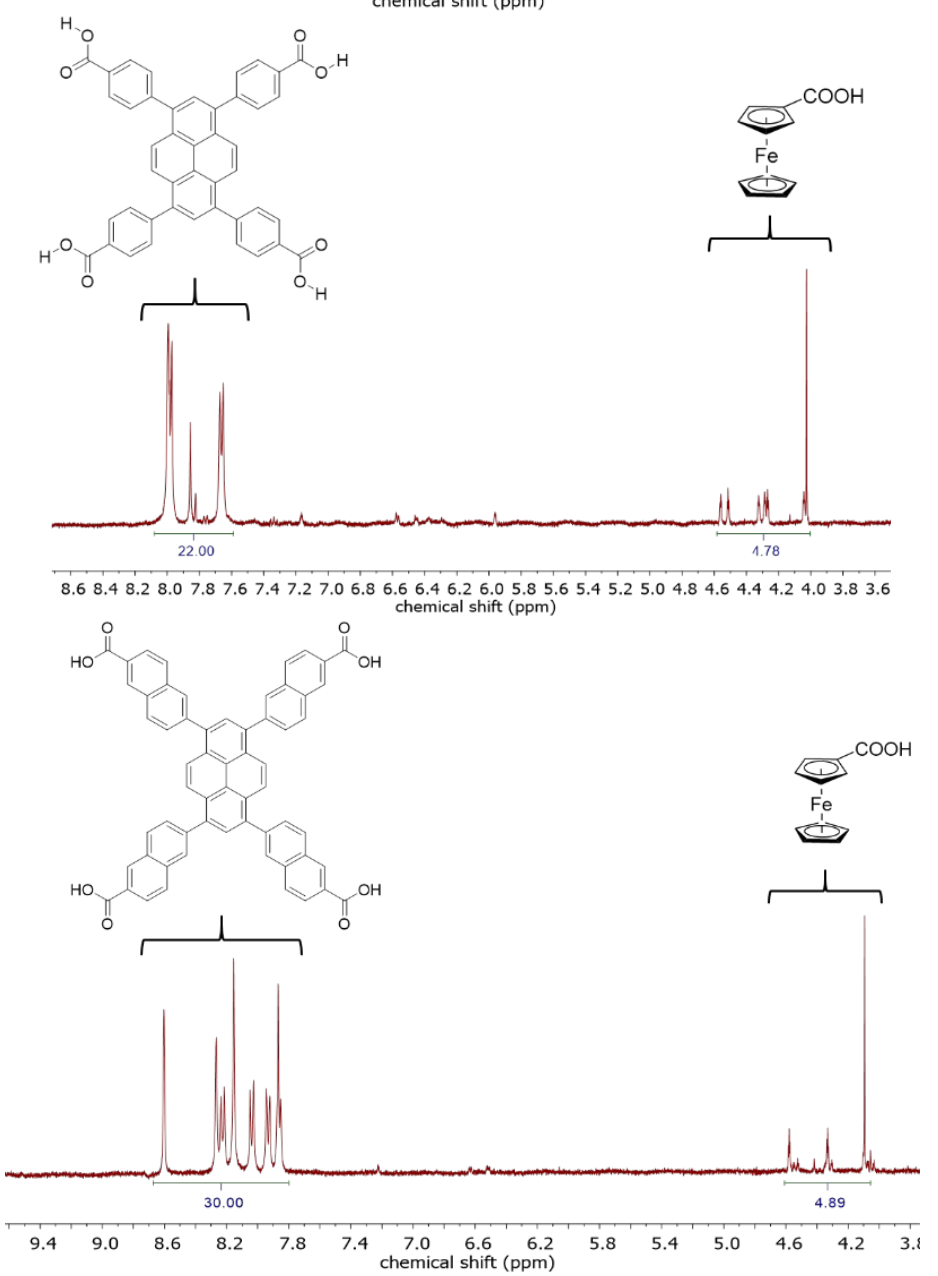

Figure S1. ${ }^{1} \mathrm{H}$ NMR spectra of digested Fc-MOF-808 (top), Fc-NU-1000 (middle), and Fc-NU1003 (bottom). 
Section S3. $\mathrm{N}_{2}$ sorption isotherm studies
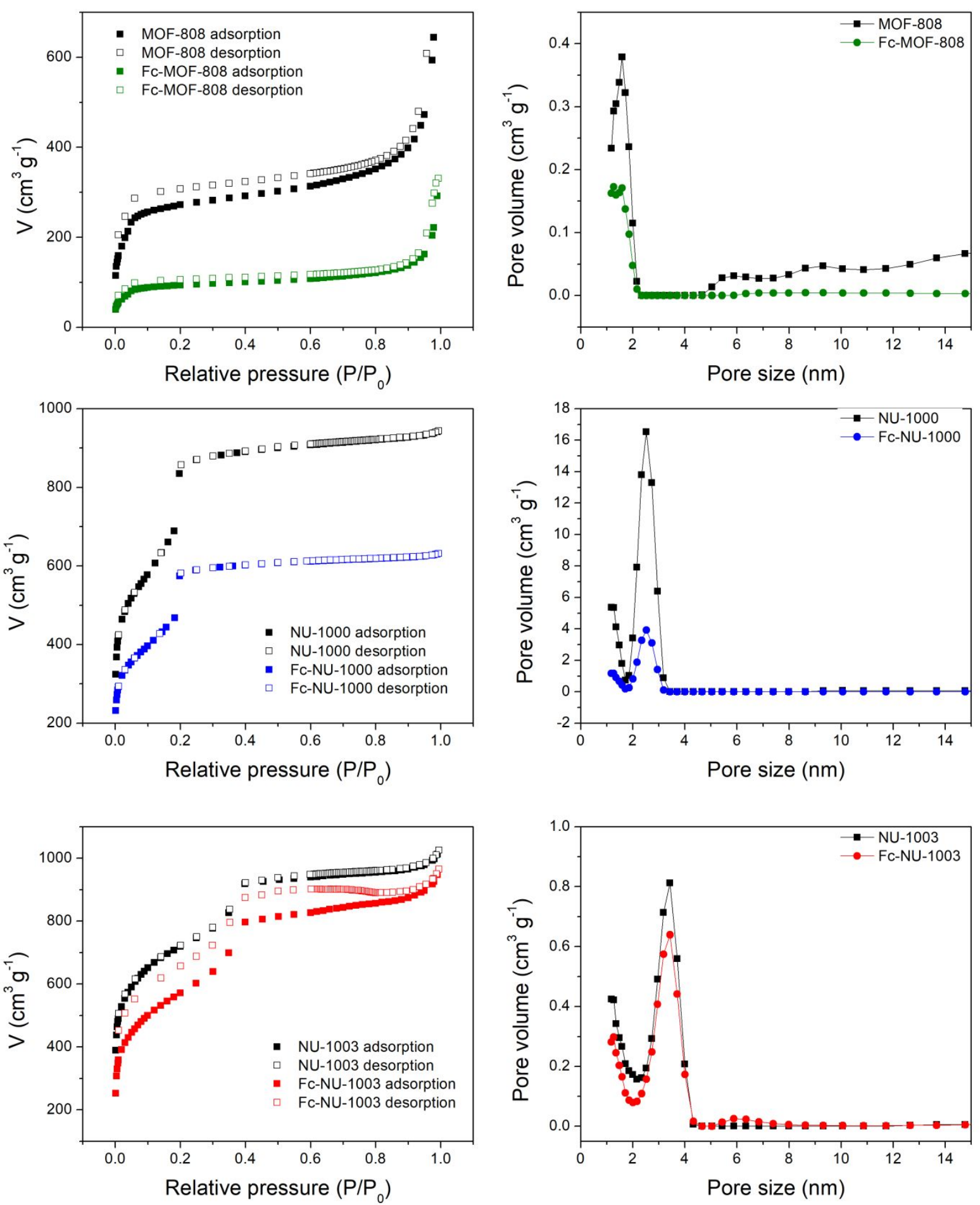

Figure S2. (left) $\mathrm{N}_{2}$ sorption isotherms and (right) pore size distribution of Fc-MOF-808 and MOF-808 (top), Fc-NU-1000 and NU-1000 (middle), and Fc-NU-1003 and NU-1003 (bottom) 
BET analysis

The BET surface areas of three Fc-MOFs and parent MOFs were calculated according to a reported method, and summarized in Table S1.

Table S1. BET surface areas of Fc-MOF-808, Fc-NU-1000, and Fc-NU-1003.

\begin{tabular}{l|cc}
\hline & BET surface area $\left(\mathrm{m}^{2} \mathrm{~g}^{-1}\right)$ & Pore size $(\mathrm{nm})$ \\
\hline Fc-MOF-808 & 390 & 1.59 \\
Fc-NU-1000 & 1690 & 2.52 \\
Fc-NU-1003 & 2032 & 3.43 \\
MOF-808 & 1136 & 1.59 \\
NU-1000 & 2508 & 2.52 \\
NU-1003 & 2627 & 3.43 \\
\hline
\end{tabular}

The parent NU-1000 and NU-1003 exhibited similar BET surface areas as reported previously (2315 $\mathrm{m}^{2} \mathrm{~g}^{-1}$ for $\mathrm{NU}-1000^{7}$ and $2700 \mathrm{~m}^{2} \mathrm{~g}^{-1}$ for NU-10032). MOF-808 exhibited a smaller BET surface area than literature $\left(2020 \mathrm{~m}^{2} \mathrm{~g}^{-1}\right)^{8}$, probably due to the remaining modulator formic acid molecules. Compared to three parent MOFs, Fc-MOFs exhibited smaller BET surface areas. 
Section S4. Scanning electron microscopy (SEM) images

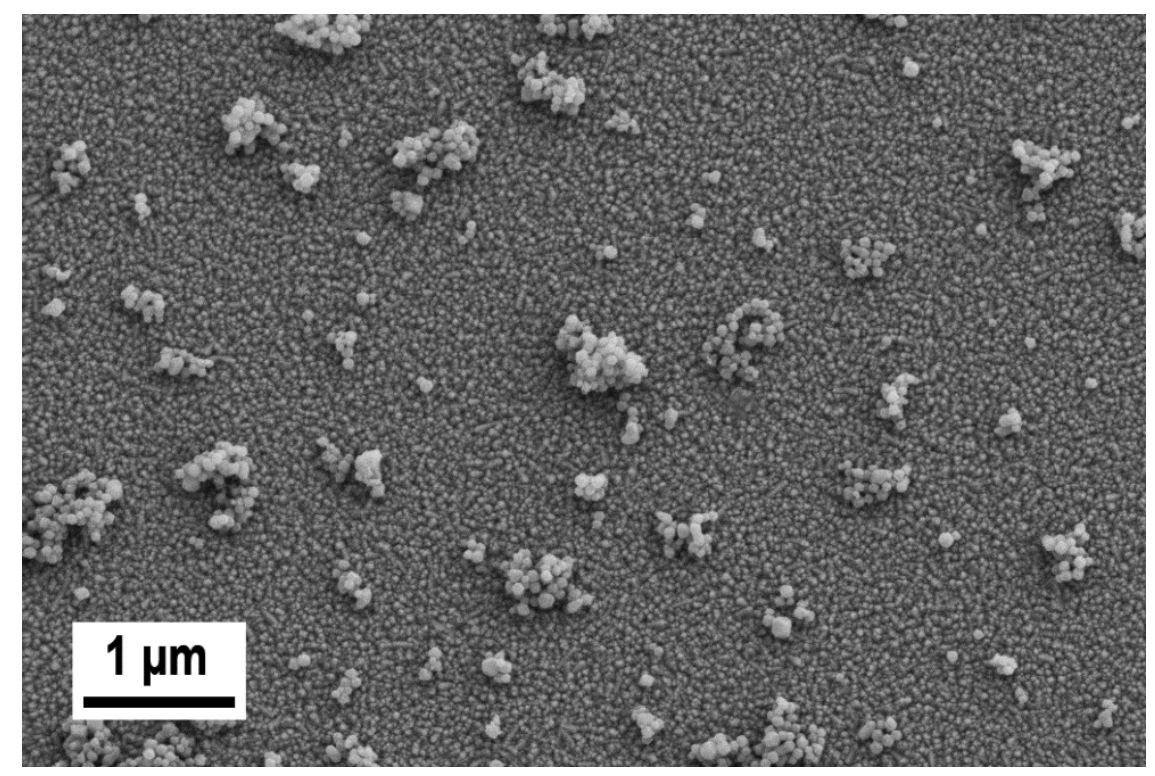

Figure S3. SEM image of Fc-MOF-808 film

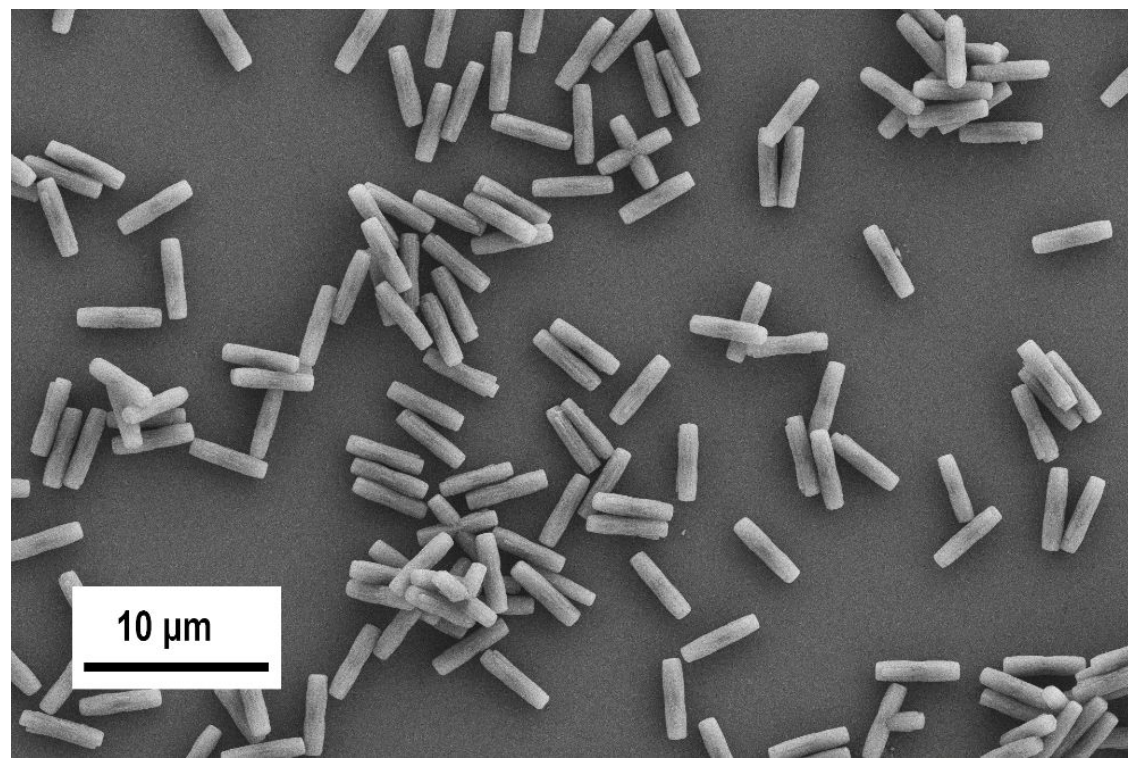

Figure S4. SEM image of Fc-NU-1000 


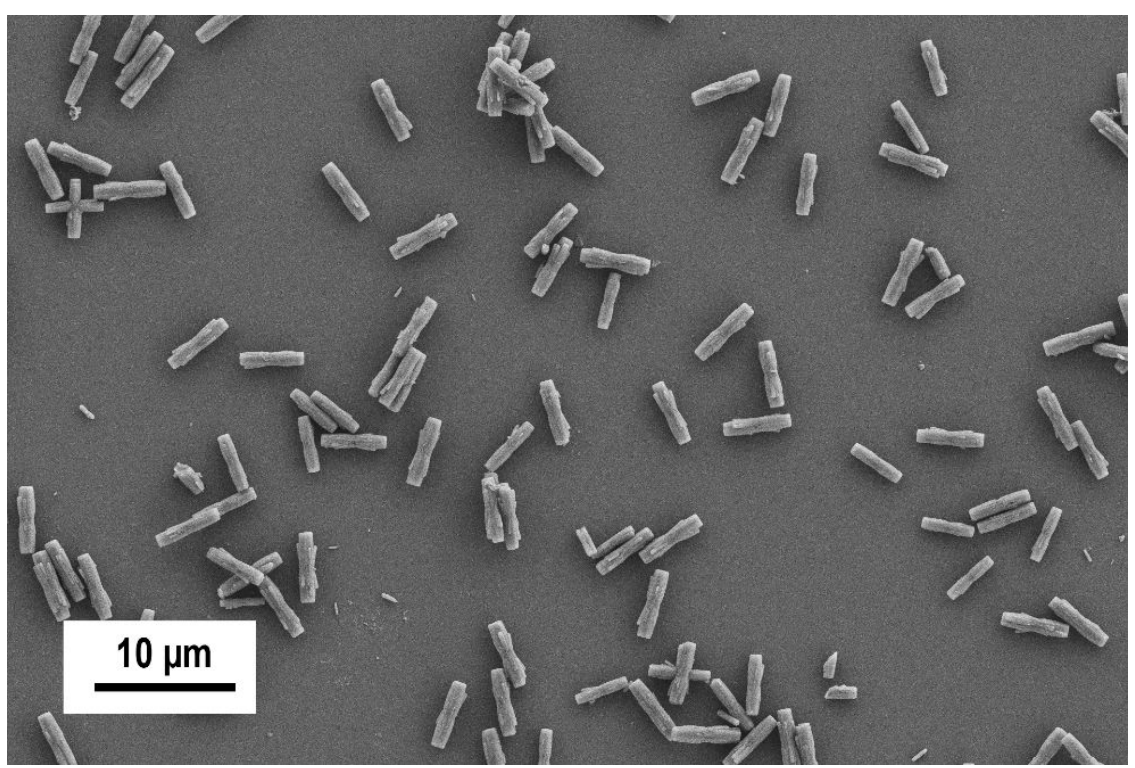

Figure S5. SEM image of Fc-NU-1003 
Section S5. Scan rate dependence studies
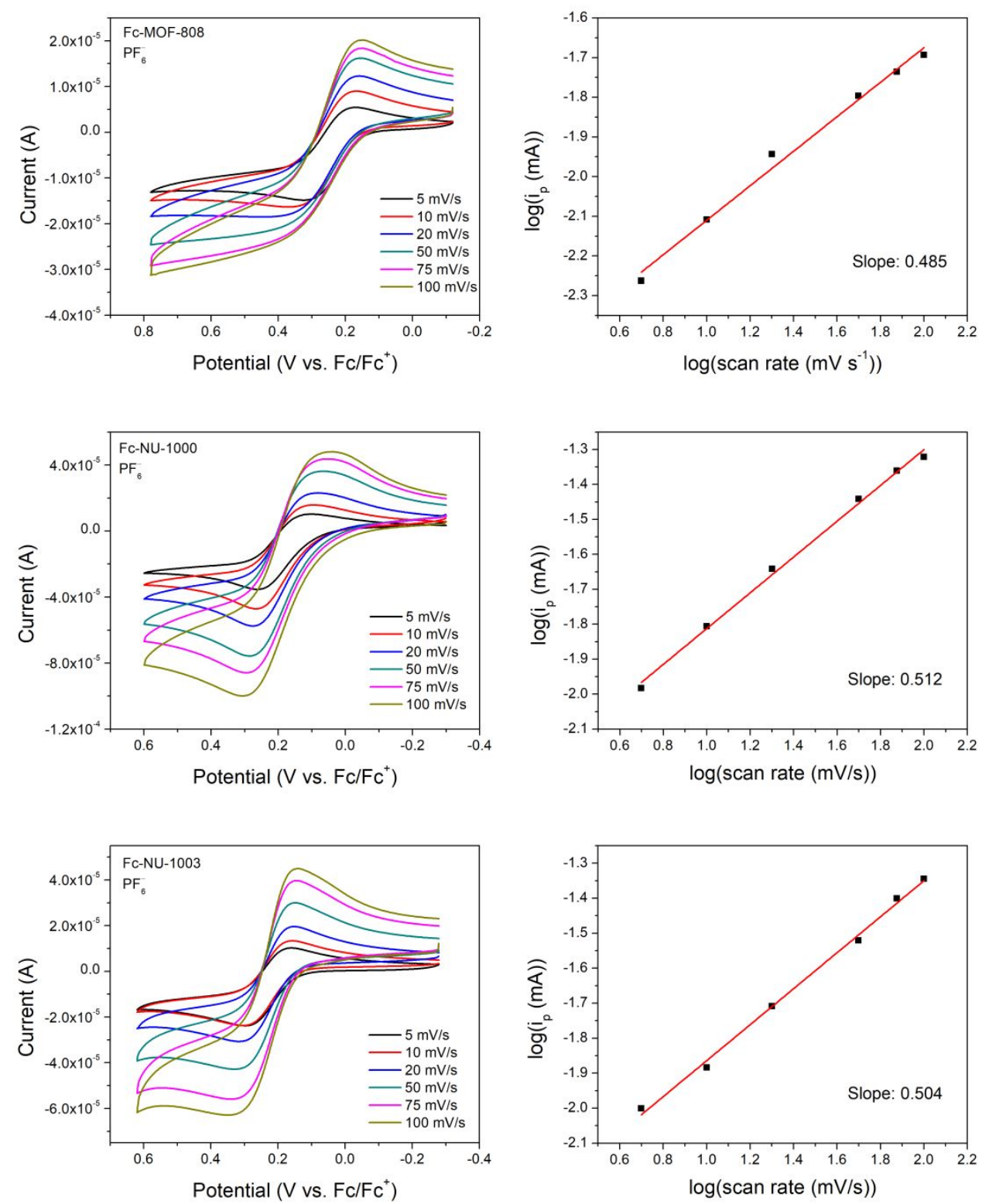

Figure S6. (left) CVs of Fc-MOF-808 (top), Fc-NU-1000 (middle), and Fc-NU-1003 (bottom) at different scan rates $(5-100 \mathrm{mV} / \mathrm{s})$; (right) the logarithms of the peak current vs. the logarithms of scan rates. 
In redox hopping, charges can hop from one electroactive site $(\mathrm{Fc})$ to another, leading to a diffusion-controlled current behavior that follows the well-known Randles-Sevcik equation:

$$
i_{p}=0.4463 n F A C\left(\frac{n F v D}{R T}\right)^{1 / 2}
$$

where $i_{p}$ is the peak current, $n$ is the number of electrons transferred in the redox reaction, $F$ is the Faraday constant, $A$ is the electrode area, $C$ is the concentration of the electroactive species, $v$ is the scan rate, $D$ is the diffusion coefficient, $\mathrm{R}$ is gas constant, and $\mathrm{T}$ is temperature.

This equation suggests a linear dependence of $\log \left(i_{p}\right)$ on $\log (v)$ with a slope of 0.5 .

As shown in Figure S6, three Fc-MOFs all demonstrated a linear dependence of the peak currents on scan rates that obeys Randles-Sevcik equation. 
Section S6. Quantification of $D_{e}$ and $D_{i}$

As shown in Figure 3, current-time responses of each Fc-MOF film were collected. According to the theoretical model, ${ }^{9}$ the conversion of the MOF microcrystal begins with a short initial reaction period when electrons diffused rapidly along the crystalsolution boundary (stage A), followed by a second period when electrons and ions migrate from the interface into the crystal bulk (stage B). These two stages are distinguished by the moment $\left(t_{r e f}\right)$ when the conversion along the crystal-electrolyte boundary is accomplished. To determine $t_{r e f} I \sqrt{t}$ vs $\sqrt{t}$ responses were plotted using chronoamperometry data, as shown in Figure S7.

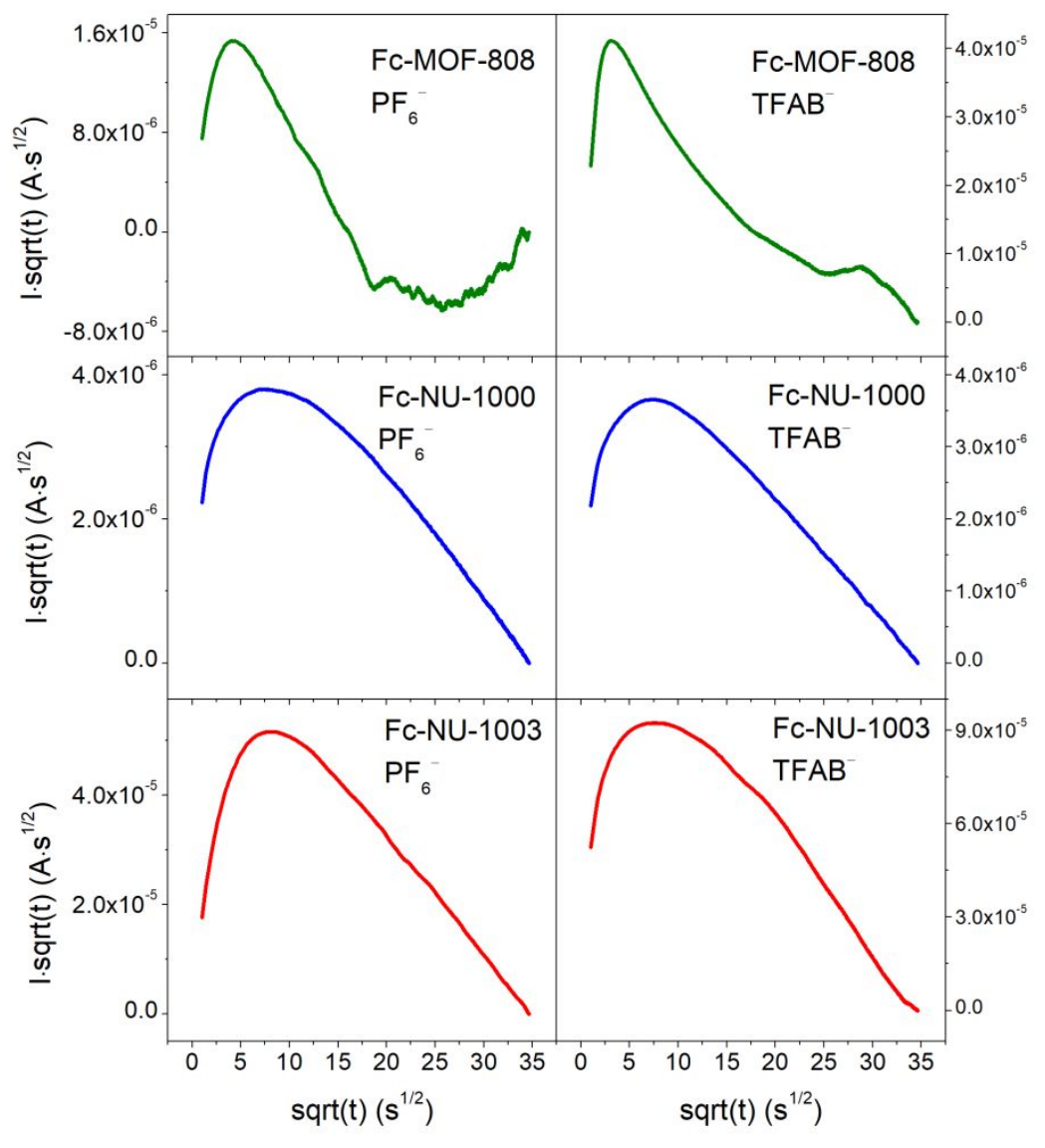


Figure S7. $I \sqrt{ } t$ vs $\sqrt{ } t$ responses for Fc-MOF-808 (green), Fc-NU-1000 (blue), and Fc-NU1003 (red) using $\mathrm{PF}_{6}^{-}$(left) or TFAB- as electrolyte.

The maximum point in each $I \sqrt{ } t$ vs $\sqrt{t}$ response was the moment distinguishing stage A and B. For instance, for Fc-MOF-808 in $\mathrm{PF}_{6}{ }^{-}$(left green curve in Figure S5), maximum ${ }_{t}$ is $4.3 \mathrm{~s}^{1 / 2}$. Therefore, $t_{\text {ref }}$ is $4.3^{2}=18.6 \mathrm{~s}$.

\section{Stage A.}

During stage A, current vs time response should follow:

$$
I(t)=\frac{N F}{V_{m}}\left[\frac{1}{1+\exp (-\varphi)}\right]\left[u\left(\frac{\Delta x_{0} \sqrt{D_{e}}+\Delta z_{0} \sqrt{D_{i}}}{2 \sqrt{\pi t}}+\sqrt{D_{e} D_{i}}\right)-4 D_{i} \sqrt{2 D_{e} t}\right]
$$

where $\varphi$ is defined as:

$$
\varphi=\frac{F}{R T}\left(E-E_{f}\right)
$$

Parameters in eq. (S1) and (S2) are defined as:

\begin{tabular}{ll}
\hline$F$ & Faraday's constant, $=96485 \mathrm{C} \mathrm{mol}^{-1}$ \\
$R$ & gas constant, $=8.314 \mathrm{~J}(\mathrm{~mol} \cdot \mathrm{K})^{-1}$ \\
$T$ & Temperature, $=298 \mathrm{~K}$ \\
$N$ & number of MOF crystallites in the film \\
$V_{m}$ & molar volume of MOF \\
$E_{f}$ & formal potential; $E_{1 / 2}$ from CVs \\
$E$ & applied potential $\left(E_{2}\right)$ \\
\hline
\end{tabular}




\begin{tabular}{ll}
\hline$u$ & perimeter of three-phase junction \\
$\Delta x_{0}$ & distance an ion travels in one hop \\
$\Delta z_{0}$ & distance an electron travels in one hop \\
\hline
\end{tabular}

$\phi$ was determined according to eq. (S2).

Table S2. Determination of $\phi$.

\begin{tabular}{l|ccc}
\hline & MOF-808 & NU-1000 & NU-1003 \\
\hline$E_{f}\left(E_{1 / 2}\right)(\mathrm{V}$ vs Fc/Fc $)$ & $0.217 \pm 0.007$ & $0.216 \pm 0.001$ & $0.238 \pm 0.001$ \\
$E_{2}(\mathrm{~V}$ vs Fc/Fc $)$ & 0.716 & 0.515 & 0.549 \\
$\phi$ & 19.43 & 11.64 & 12.11 \\
\hline
\end{tabular}

$V_{m}$ was calculated by $V_{m}=\frac{\text { molar mass }}{\text { density }}$. Molecular weights were calculated from MOF formulas. Densities of MOF-808 and NU-1000 were from reference. ${ }^{10}$ Density of NU1003 was calculated on http://checkcifiucr.org, a service of the International Union of Crystallography.

Table S3. Calculation of molar volume.

\begin{tabular}{l|ccc}
\hline & MOF-808 & NU-1000 & NU-1003 \\
\hline Molecular weight $\left(\mathrm{g} \mathrm{mol}^{-1}\right)$ & 1362 & 2180 & 2576 \\
Density $\left(\mathrm{g} \mathrm{cm}^{-3}\right)$ & 0.955 & 0.473 & 0.304 \\
\hline
\end{tabular}




\begin{tabular}{l|lll}
\hline Molar volume $\left(\mathrm{cm}^{3} \mathrm{~mol}^{-1}\right)$ & 1428.178 & 4609.598 & 8473.684 \\
\hline
\end{tabular}

$u$ is the perimeter of the surface of each MOF crystallite that was immobilized on the FTO. For NU-1000 and NU-1003, $u$ is $2 \times($ length+width); for MOF-808, each octahedron was approximately considered as a cube with equal side length. The width/side length was also used as the height $H$ for calculation in stage B. Length and width can be measured from SEM images using software ImageJ following a previous report. ${ }^{6}$

Table S4. Calculation of $u$.

\begin{tabular}{l|ccc}
\hline & MOF-808 & NU-1000 & NU-1003 \\
\hline Length $(\mathrm{cm})$ & $0.498 \times 10^{-4}$ & $3.861 \times 10^{-4}$ & $5.154 \times 10^{-4}$ \\
Width/Height $(\mathrm{cm})$ & $0.498 \times 10^{-4}$ & $0.833 \times 10^{-4}$ & $1.113 \times 10^{-4}$ \\
$u(\mathrm{~cm})$ & $1.992 \times 10^{-4}$ & $9.388 \times 10^{-4}$ & $12.53 \times 10^{-4}$ \\
\hline
\end{tabular}

$N$ was determined based on the total charge $Q_{t}$ that passed during reaction. The total number of Fc, $n$, in one film is $Q_{t} / \mathrm{F}$, where $\mathrm{F}$ is Faraday's constant. The number of $\mathrm{F}_{\mathrm{c}}$ in one MOF particle in moles, which is equivalent to the number of nodes on one MOF particle, can be calculated by $\frac{\text { volume of each MOF particle }}{\text { molar volume }} . N=\frac{n}{\text { number of Fc in one MOF particle }} . N$ was calculated and used respectively for each MOF film. 
$\Delta x_{0}$ and $\Delta z_{0}$ were determined by measuring the Fc-to-Fc (i.e. node-to-node) distance along the direction that an electron/ion hops. For instance, an illustration of NU-1000 MOF microcrystal immobilized on FTO was shown in Figure S8. According to the model, electrons hop along $z$ direction whereas ions can diffuse from the surfaces into the bulk of MOF microcrystal along $x$ and $y . \Delta z_{0}$ was measured to be $20.839 \AA$. An average of hopping distances in $x$ and $y$ directions was used as ion hopping distance, $\Delta x_{0}$.
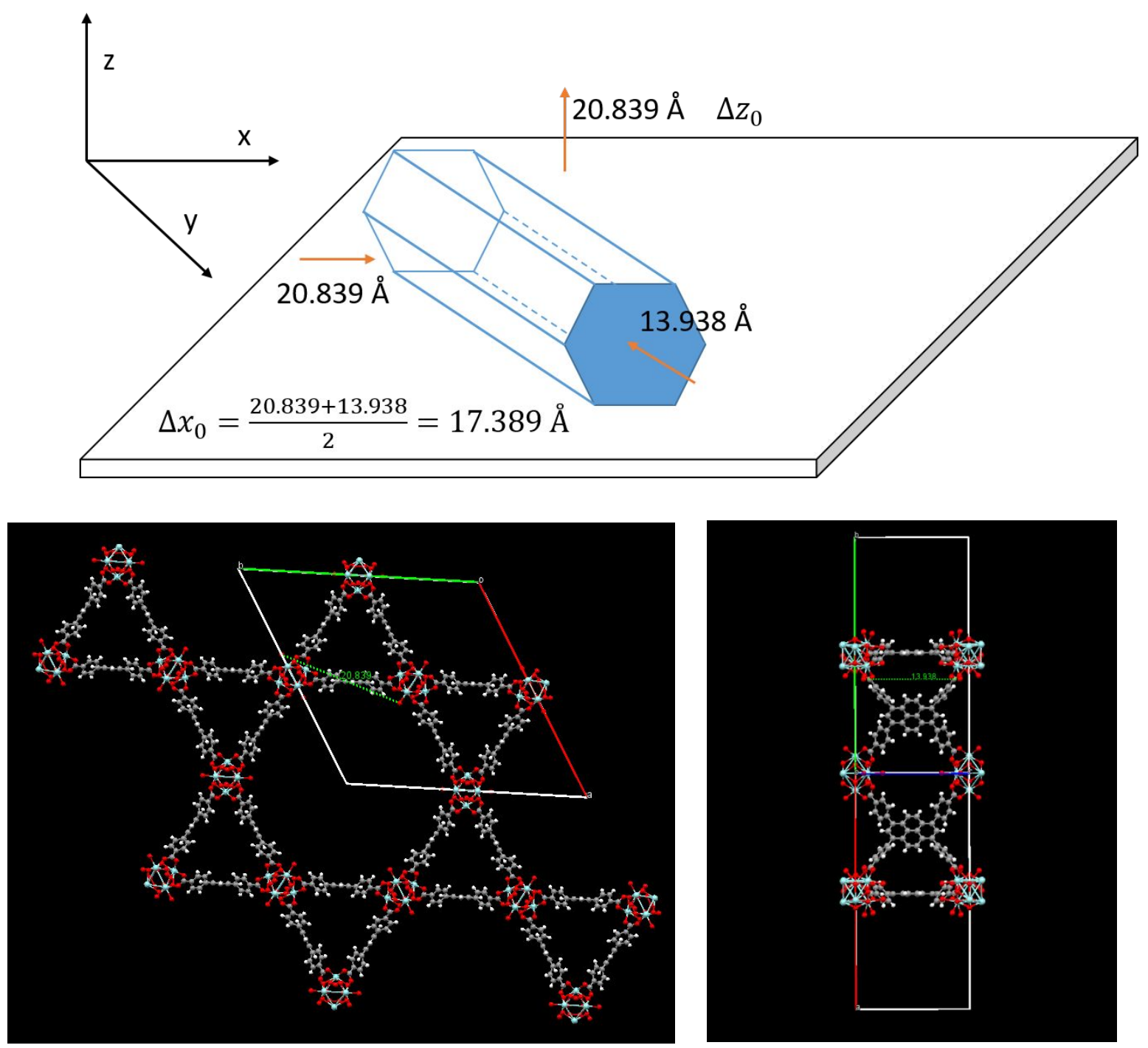
Figure S8. (top) Illustration of one NU-1000 MOF microcrystal on FTO. (bottom) hopping distance along $z$ or $x$ (left) and hopping distance along $y$ (right).

Table S5. $\Delta x_{0}$ and $\Delta z_{0}$ for three MOFs.

\begin{tabular}{l|ccc}
\hline & MOF-808 & NU-1000 & NU-1003 \\
\hline$\Delta x_{0}(\mathrm{~cm})$ & $1.427 \times 10^{-7}$ & $1.739 \times 10^{-7}$ & $2.244 \times 10^{-7}$ \\
$\Delta z_{0}(\mathrm{~cm})$ & $1.427 \times 10^{-7}$ & $2.084 \times 10^{-7}$ & $2.647 \times 10^{-7}$ \\
\hline
\end{tabular}

After determination of parameters, eq. (S1), an expression of $I$ as a function of $D_{e}$ and $D_{i}$ was fitted to $I$ vs $t$ responses from $t=0$ to $t=t_{\text {ref }}$ (black curves in Figure S9). $D_{e}$ and $D_{i}$ can be obtained.

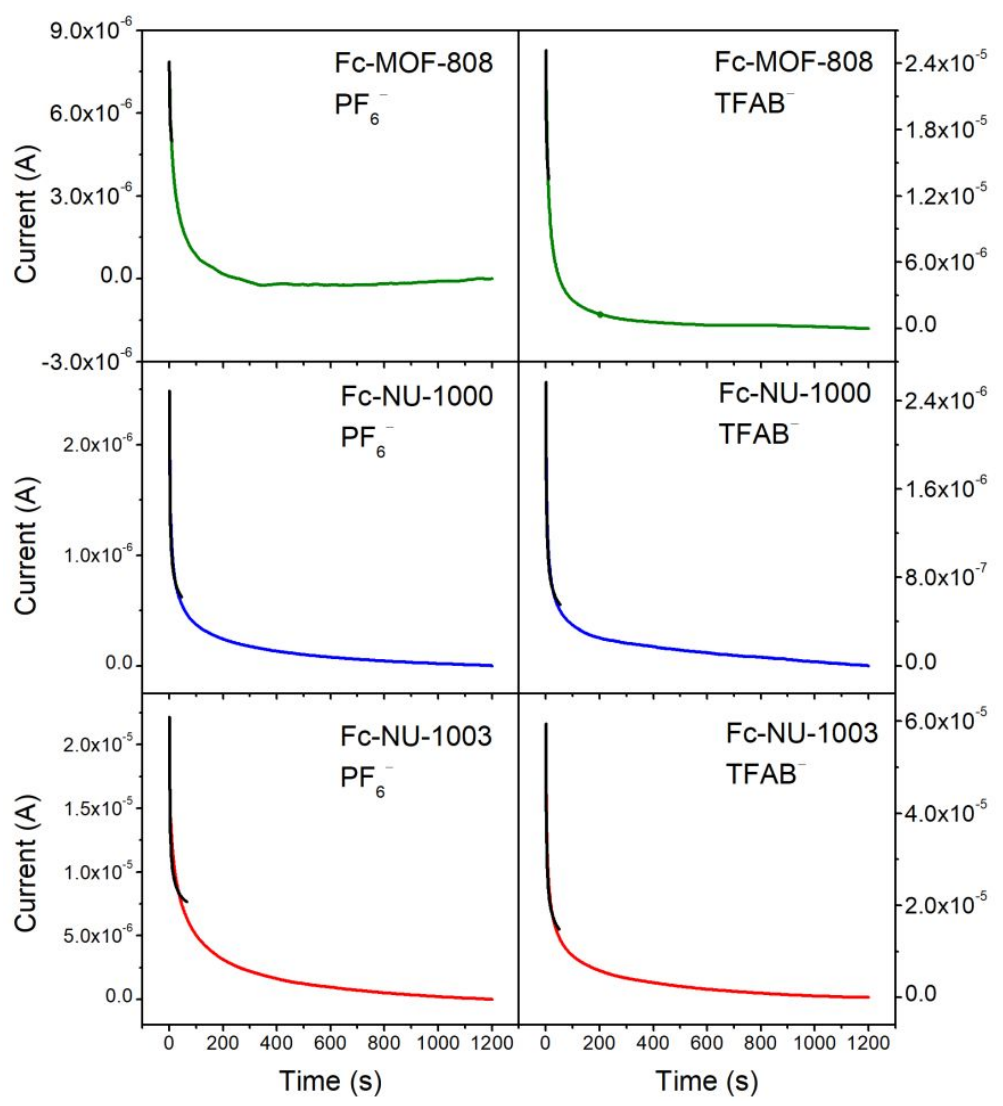


Figure S9. CAs with black curves showing fitting results of Fc-MOF-808 (green), and FcNU-1000 (blue), and Fc-NU-1003 (red) using 0.1 M TBAPF 6 (left) or TBATFAB (right) as electrolyte.

\section{Discussion of Fc-MOF-808}

As illustrated in Figure 4 in the paper, the theoretical model was developed for an ideal cuboid microcrystal with one rectangle surface in direct contact with the substrate. ${ }^{6}$ Both NU-1000 and NU-1003 particles are in the shape of hexagonal column that resembles a cuboid, and they are immobilized on FTO slides with a rectangle shape contact. However, MOF-808 microcrystals are octahedral. The contacting interface between a MOF- 808 particle and the FTO slide is triangular. Therefore, the three dimensions $(\mathrm{x}, \mathrm{y}$, and $\mathrm{z})$ defined by the model for a cuboid microcrystal may lose validity when applied to MOF-808. Recently, Goswami et al. reported anisotropic redox conductivity in NU-1000. The charge hopping coefficient was found to differ by a factor of up to 3500 times along different diffusion directions. ${ }^{11}$ This work further highlights the importance of MOF morphology in determining charge hopping efficiency. Moreover, due to smaller particle sizes, more aggregation is observed in Fc-MOF-808 films (Figure S3), which results in essentially larger aggregated MOF particles. As mentioned above, the theoretical model was developed for one single ideal microcrystal. Given that each Fc-MOF-808 microcrystal in the aggregate is in direct contact with the surrounding ones, they may no longer be considered as individually 
separate microcrystals. The interference from neighboring MOF microcrystals needs to be accounted for to apply the model.

\section{Stage B.}

As discussed in the paper, the moment $\left(t_{r e f}\right)$ is the beginning of stage B, when electrons and ions start to migrate from the interface to the bulk of MOF microcrystals. $D_{e}$ and $D_{i}$ satisfy eq. (4) and eq. (5) in the paper. $H$ is the height of MOF film, equivalent to the height/width of each MOF particle (Table S4). $N$ and $u$ were determined in stage A. Therefore, $D_{e}$ and $D_{i}$ can be calculated. $D_{e}$ and $D_{i}$ values in Table 1 in the paper are average of calculations of three trials under same conditions. 
Section S7. Calculation of $D_{a p p}$ based on Anson plots

The charge $Q$ that passed after certain reaction time can be calculated by integration of current over time. Anson plots, i.e. charge vs square-root-of-time responses for each FcMOF thin film can be obtained, as shown in Figure S10.

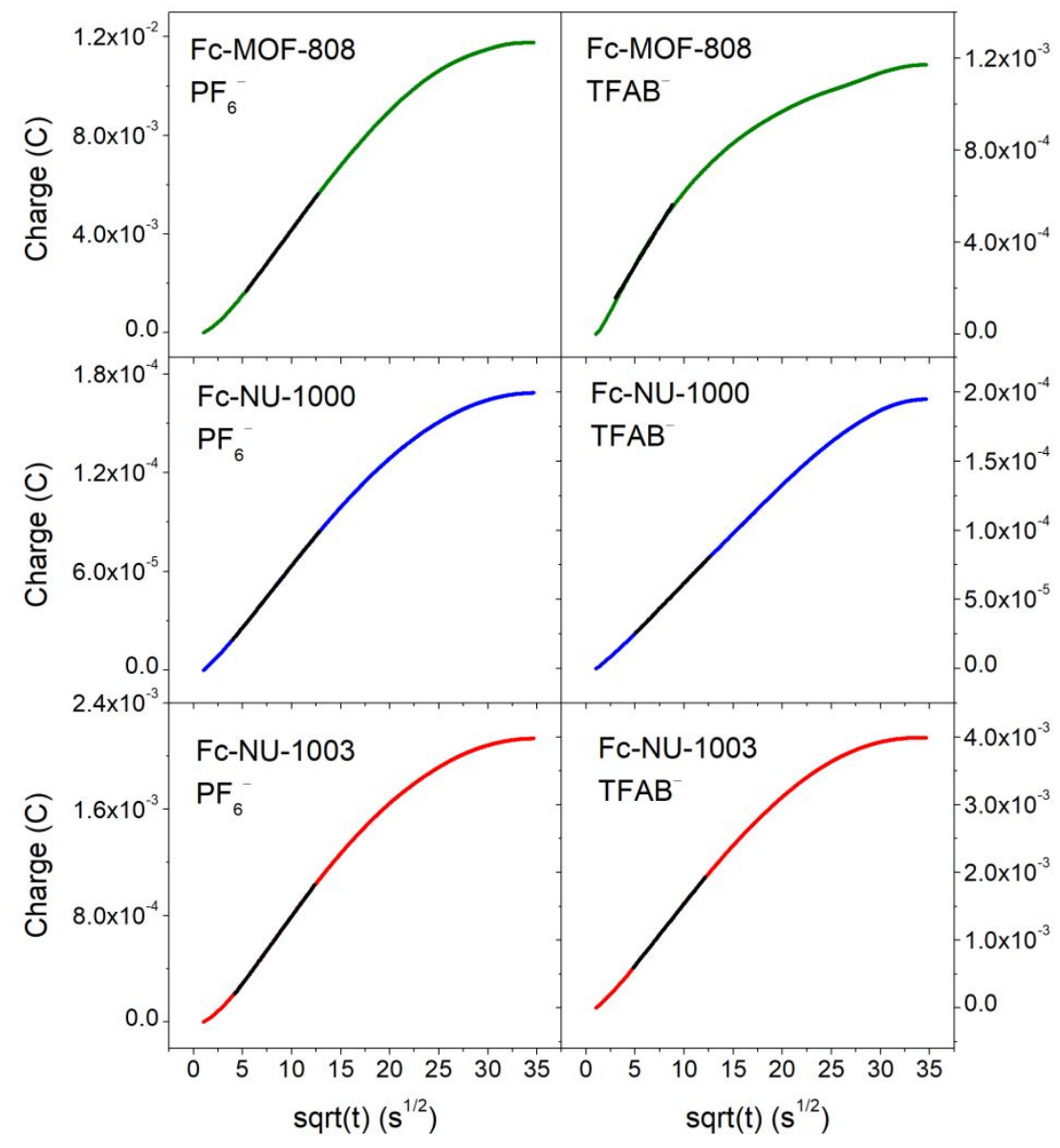

Figure S10. Anson plots (charge vs square-root-of-time) for Fc-MOF-808 (green), Fc-NU-1000 (blue), and Fc-NU-1003 (red) using $\mathrm{PF}_{6}{ }^{-}$(left) or $\mathrm{TFAB}^{-}$as electrolyte. Black curves: linear fitting.

According to Anson equation: 


$$
Q=2 n F A C D_{a p p}^{1 / 2} t^{1 / 2} \pi^{1 / 2}
$$

where $Q$ is the overall charge that passes during the electrolysis time, $n$ is the number of electrons transferred in the redox reaction, $F$ is the Faraday constant, $A$ is the electrode area, $C$ is the concentration of the electroactive species, $D_{a p p}$ is the apparent diffusion coefficient, and $t$ is the time.

$C$, the concentration of the electroactive species, can be calculated following:

$$
C=\frac{n}{V}=\frac{Q_{t} / F}{A \times d}
$$

where $n$ is the number of electroactive species $(\mathrm{Fc}), V$ is the total volume of the MOF thin film, $Q_{t}$ is the total charge passing during reaction, and $d$ is the thickness of MOF film (i.e. the height of MOF microcrystals, which can be measured from SEM images).

By plugging eq. (S4) into eq. (S3), $D_{a p p}$ can be calculated by:

$$
D_{\text {app }}=\left(\frac{\text { slope } \times \sqrt{\pi} \times d}{2 \times Q_{t}}\right)^{2}
$$

where slope is the slope $\left(Q / t^{1 / 2}\right)$ of Anson plots, obtained by linear fitting.

\section{Example calculation for $\mathrm{Fc}-\mathrm{NU}-1000$ using $\mathrm{PF}_{6}{ }^{-}$as electrolyte}

The overall charge $\left(Q_{t}\right)$ that passed during 20 min of reaction time is $1.69 \times 10^{-4} \mathrm{C}$, and the slope of Anson plot of Fc-NU-1000 using $\mathrm{PF}_{6}^{-}$(left blue curve in Figure $\mathrm{S} 10$ ) is $7.48 \times 10^{-6} \mathrm{C} \mathrm{s}^{-1 / 2}$. The thickness of Fc-NU-1000 is $\sim 0.833 \mu \mathrm{m}$ (= the width of Fc-NU-1000 microcrystals, determined from Figure S4). Therefore, $D_{a p p}$ was calculated to be $1.08 \times 10^{-11} \mathrm{C} \mathrm{cm}^{2} \mathrm{~s}^{-1}$. Each $D_{a p p}$ was the average of calculations of three films under same conditions. 


\section{Measurement of capacitive current contribution}

It is noteworthy to mention that capacitive current can contribute to the obtained chronoamperometry data. To measure how significant the contribution from the capacitive current would affect the overall current response, we collected chronoamperometry data from an unmodified NU-1000 film under same conditions as for Fc-NU-1000 films. As shown in Figure S11, compared to the response of Fc-NU-1000 (blue line), unmodified NU-1000 (black line) has a much smaller current response. The current difference (red dotted line) is very close to the current response of Fc-NU-1000. Therefore, in our case, the capacitive current has little contribution to the obtained data. As a result, the capacitive current was not excluded from the data in calculations. 


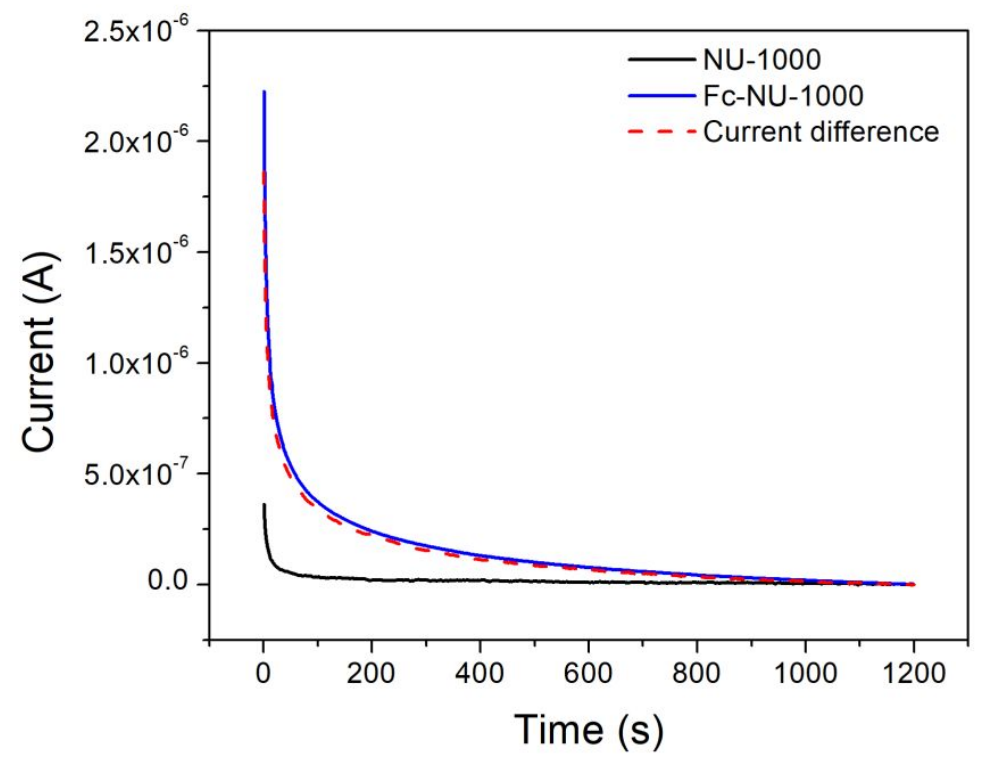

Figure S11. Current vs. time responses of Fc-NU-1000 (blue line) and NU-1000 (black line); the current difference between the two is shown as red dotted line. 


\section{Section S8. References}

(1) Mondloch, J. E.; Bury, W.; Fairen-Jimenez, D.; Kwon, S.; DeMarco, E. J.; Weston, M. H.; Sarjeant, A. A.; Nguyen, S. T.; Stair, P. C.; Snurr, R. Q. Vapor-phase metalation by atomic layer deposition in a metal-organic framework. J. Am. Chem. Soc. 2013, 135, 10294-10297.

(2) Li, P.; Moon, S.-Y.; Guelta, M. A.; Lin, L.; Gómez-Gualdrón, D. A.; Snurr, R. Q.; Harvey, S. P.; Hupp, J. T.; Farha, O. K. Nanosizing a Metal-Organic Framework Enzyme Carrier for Accelerating Nerve Agent Hydrolysis. ACS Nano 2016, 10, 9174-9182.

(3) LeSuer, R. J.; Buttolph, C.; Geiger, W. E. Comparison of the conductivity properties of the tetrabutylammonium salt of tetrakis (pentafluorophenyl) borate anion with those of traditional supporting electrolyte anions in nonaqueous solvents. Anal. Chem. 2004, 76, 6395-6401.

(4) Hod, I.; Bury, W.; Gardner, D. M.; Deria, P.; Roznyatovskiy, V.; Wasielewski, M. R.; Farha, O. K.; Hupp, J. T. Bias-switchable permselectivity and redox catalytic activity of a ferrocene-functionalized, thin-film metal-organic framework compound. J. Phys. Chem. Lett. 2015, 6, 586-591.

(5) Shearer, G. C.; Chavan, S.; Bordiga, S.; Svelle, S.; Olsbye, U.; Lillerud, K. P. Defect engineering: tuning the porosity and composition of the metal-organic framework UiO-66 via modulated synthesis. Chem. Mater. 2016, 28, 3749-3761.

(6) Celis-Salazar, P. J.; Cai, M.; Cucinell, C.; Ahrenholtz, S. R.; Epley, C. C.; Usov, P. M.; Morris, A. J. Independent Quantification of Electron and Ion Diffusion in Metallocene-Doped Metal-Organic Frameworks Thin Films. J. Am. Chem. Soc. 2019, 141, 11947-11953.

(7) Deria, P.; Bury, W.; Hupp, J. T.; Farha, O. K. Versatile functionalization of the NU-1000 platform by solvent-assisted ligand incorporation. Chem. Commun. 2014, 50, 1965-1968.

(8) Furukawa, H.; Gándara, F.; Zhang, Y.-B.; Jiang, J.; Queen, W. L.; Hudson, M. R.; Yaghi, O. M. Water adsorption in porous metal-organic frameworks and related materials. J. Am. Chem. Soc. 2014, 136, 4369-4381.

(9) Schröeder, U.; Oldham, K. B.; Myland, J. C.; Mahon, P. J.; Scholz, F. Modelling of solid state voltammetry of immobilized microcrystals assuming an initiation of the electrochemical reaction at a three-phase junction. J. Solid State Electrochem. 2000, 4, 314-324.

(10) Drout, R. J.; Howarth, A. J.; Otake, K.-i.; Islamoglu, T.; Farha, O. K. Efficient extraction of inorganic selenium from water by a $\mathrm{Zr}$ metal-organic framework: investigation of volumetric uptake capacity and binding motifs. CrystEngComm 2018, 20, 6140-6145.

(11) Goswami, S.; Hod, I.; Duan, J.; Kung, C.-W.; Rimoldi, M.; Malliakas, C. D.; Palmer, R. H.; Farha, O. K.; Hupp, J. T. Anisotropic Redox Conductivity within a Metal-Organic Framework Material. $J$. Am. Chem. Soc. 2019, 141, 17696-17702. 